\title{
Treatment for Colorectal Cancer: Robot-assisted vs Laparoscopic Surgery
}

\author{
Shivani Kamlesh Modi and Satyajit Patra* \\ American International Medical University, Gros Islet, Saint Lucia \\ *Corresponding author: Satyajit Patra, American International Medical University, Gros Islet, Saint Lucia \\ To Cite This Article: Satyajit Patra. Treatment for Colorectal Cancer: Robot-assisted vs Laparoscopic Surgery. Am J Biomed Sci \& Res. 2019 - 4(6). \\ AJBSR.MS.ID.000856. DOI: 10.34297/AJBSR.2019.04.000856
}

Received: 眥 July 26, 2019; Published: 笽 August 28, 2019

\begin{abstract}
Over the past few years, Robotic surgery has been an emerging field in colorectal surgery. Over years there has been continuous shift towards minimally invasive procedures with enormous potential advantages, but progress is impeded because of limited evidence, lack of technology and cost of expenditure. However, recent advances and feedback in robotic surgery and single-port incision in laparoscopic surgery are likely to improve surgical outcomes for treatment of colorectal cancer. Currently, laparoscopic surgery is preferred for colorectal cancer over open surgery and its usefulness is unquestionable. Laparoscopic surgery has become the standard of practice for various digestive tract surgeries. Although, robotassisted is gold standard for various other procedures, more research is needed to prove its safety and efficacy in the treatment of colorectal cancer. Robotic surgery may overcome limitations of laparoscopic surgery such as assistant dependent camera movements, retraction issues, lack of userfriendly environment and rigid instrumentation. Prolonged operative time, learning time, increased expenses and more trauma to the patient and family are major drawbacks of robotic surgery. In recent years robot-assisted laparoscopic surgery has been increasingly applied, again with lack of comparison and evidence over conventional laparoscopic surgery. The aim of this study is to compare robotic-assisted surgery and laparoscopicassisted surgery for the treatment of colorectal cancer (CRC).
\end{abstract}

Keywords: Colorectal cancer; Complication; Estimated blood loss; Length of hospital stay; Network meta-analysis; Operation time

\section{Introduction}

Colorectal malignant growth is a disease that begins in the colon or the rectum. These malignant growths can likewise be named colon disease or rectal malignant growth, contingent upon where they begin. Colon malignant growth and rectal disease are regularly gathered together collectively as they share same features. CRC is the third most generally analysed malignant growth in men and the second in women, with 1.8 million new cases and very nearly 861,000 deaths in 2018 as indicated by the World Health Organization. Throughout the decade, open medical procedure was considered the far reaching and objective standard for treatment and medical procedure of colorectal malignant growth. The point of careful treatment is tumour size, lymphatic waste, lymph node resection alongside clear careful edges. [1-6] In recent years there have been changes towards minimally invasive procedures and techniques, including the widespread adoption of laparoscopy techniques as an alternative to treat colorectal cancer instead of open surgery.

Kitano showed that contrasted and open medical procedure, laparoscopic medical procedure was a standard treatment for colon disease with shorter emergency clinic remains, quicker recuperation, improved rate of wound contamination, and diminished ago ny $[1,3,5,7-10]$. Alongside advantages comes the downsides which incorporates inflexible instrumentation, absence of representation, poor withdrawal abilities alongside camera help and absence of innovation [2]. Robotic surgery was developed to overcome the technical difficulties of conventional laparoscopy. Robotic surgery has been advantageous because of its 3-dimensional operating field, surgeon operated camera field for better visualization. In addition to that there are studies about robotic systems especially the da Vinci Xi - a robot performing colorectal surgery has been found to be better performing and more efficient over the conventional laparoscopic surgery. Almost most of the controversy surrounding the use of robotic surgery remains around the fact that robotic surgery has high expenditure and longer operative hours leading to trauma to patient and families and tiring to the doctor as well. However, further advancement in this field has been limited by a plethora of challenges that must be addressed, including difficult implementation, and still somewhat limited technologies [10-12].

Although the limitations of laparoscopic surgery are met by robotic surgery, but the question arises whether these advantages are enough to offset the cost of robots. Furthermore, there has not 
been enough research performed over the blood loss, hospital stay, complications of the procedures and recurrence rate comparing both robotic surgery and laparoscopy surgery. The advancement in robotic surgery has leads to shorter learning curve and has been showed beneficial in various gynaecologic surgeries but its advantages in colorectal surgery are still been controversial [3,4,7,13-15]. Also, studies are very weak comparing both techniques and better clinical and surgical outcomes of the same. This investigation means to compare robotic assisted and laparoscopic ways to deal with colon and rectal methods to illustrate any distinctions in results.

\section{Methods}

\section{Data collection and analysis}

This study was a systematic review of publications in PubMed, Embase, Web of Science and Cochrane Library databases and the Cochrane Library comparing laparoscopic and robotic assisted colorectal surgery procedures. Databases were searched irrespective of dates under the Medical title "Robotic colorectal surgery" "Laparoscopic colorectal surgery" and "Robotic versus laparoscopic colorectal surgery". This study was a meta-analysis of several eligible studies from public resources, thus informed consent and approval were not necessary.

\section{Inclusion criteria}

Publications were fused into the examination in case they met the going with criteria: relative examinations taking a gander at laparoscopic versus robotic colorectal frameworks, paying little regard to type (e.g, right hemicolectomy, low principal resection, sigmoid resection); randomized controlled primers, controlled clinical fundamentals, or observational examinations, if they were close in nature; and [7] studies were included but not limited to estimated operative time, amount of blood loss, postoperative outcomes, operative complications and conversions.

\section{Exclusion criteria}

Studies were excluded if the studies had incomplete data or if the study cannot be statically analysed. Studies were also excluded on the basis if they were letter and comments. Also, the literature which included the same population demographics were also excluded. Only the literature which had complete results and include various demographics of population were included.

\section{Operative Techniques}

\section{Laparoscopic surgery}

Patient was placed in lithotomy position and general anaesthesia was induced and both arms were adjusted alongside the body. Patient was tilted about 15 degrees opposite to the side of the tumour i.e. left colon cancer patient will be tilted towards right side. The inflation of abdomen with $\mathrm{CO} 2$ is achieved (pneumoperitoneum) through inserting needle in a $1 \mathrm{~mm}$ port near umbilical area. The insufflator is set to $12-14 \mathrm{mmHg}$. A $12 \mathrm{~mm}$ port is inserted $2 \mathrm{~cm}$ below the umbilicus. Another $8 \mathrm{~mm}$ port is inserted under direct vision in the upper quadrant which will be used as Arm 1. Other two $8 \mathrm{~mm}$ ports are placed $4 \mathrm{~cm}$ away from the symphysis pubis and $5 \mathrm{~cm}$ away from the xiphoid process serving as Arm 2 and 3 respectively. One $12-\mathrm{mm}$ port is set under direct vision in the left-lower quadrant, to some degree below average contrasted with one side spin umbilical line (SUL) and insignificantly sidelong to the other side MCL. The use of inferior contrasted with preferable dissection over ligate and division the ileocolic vessels (supply course and vein), right colic vessels (conductor and vein), and right piece of the inside colic vessels (hallway and vein, as vital) [1,8, 9,11,12,16-18]. After satisfaction of get together of the ileum, cecum, rising colon, and proximal transverse colon. The precedent is isolated through this damage and is transacted. Simultaneously 12 wz, hand-sewn from beginning to end anastomosis was performed extra-corporeally $[7,8,19,20]$.

\section{Robotic surgery}

Patient was placed in lithotomy position after induction of general anaesthesia. The careful framework comprises of a control module with a top quality, three-dimensional (3D) camera where the specialist sits and controls effector mechanical arms in an "ace slave". Every framework has an endoscope and three to four effector arms that join Endo Wrist gadgets, or tradable instruments that can be controlled with more prominent mobility than the human wrist. $[6-8,15,21]$ While new frameworks are as of now being developed and will probably result in both monetary and innovation rivalry, the da Vinci remains the pioneer in mechanical careful innovation. Mechanical autonomy enables the specialist to see 3D pictures, acquire better points with the expanded degrees of opportunity given by the EndoWrist, and control three distinct instruments at the same time $[10,22]$ Laparoscopic surgery is attainable and totally tantamount to open medical procedure, with points of interest in postoperative entanglements and emergency clinic remain.

Rectal laparoscopic resection is additionally plausible, and the investigations performed to date recommend that short and longhaul results are practically identical to open medical procedure. Regardless of the upsides of the laparoscopic approach for colorectal restorative system, this procedure has a couple of limitations, for instance, loss of the 3D vision, obstacles in the open-door degrees of the cautious instruments, the escalation of the physiological tremor and the "support" sway [20,22]. Although robotic surgery provides surgeons with various advantages which make complex laparoscopic procedures like identifying important neurovascular structures and intra-corporeal suturing in a deep and narrow pelvis easier. The absence of near investigations among robotic and laparoscopic approaches in regard to explicit results, joined with the expense of new innovation like the da Vinci robot (Intuitive Surgical), is a restrictive factor for across the board selection of the robot in numerous medical clinic focuses $[3,7,8]$. 


\section{Statistical analysis}

The meta-analysis of activity time assessed blood misfortune, length of clinic remains and complexity, mortality, anastomotic spillage, wound contamination, dying, and ileus. Socioeconomics and other applicable information were gathered, including study configuration, number of patients who experienced mechanical systems, number of patients who experienced laparoscopic methods, tolerant age, weight file, quantities of people, history of stomach medical procedure, and sign for medical procedure. All information was removed from the articles' content, tables, and considers and entered along with an electronic spreadsheet for examination. For constant results, mean net contrasts (benchmark to-treatment change in treatment bunch mirrors change in charge gathering) were utilized as essential results. For clear cut results, chances proportions were utilized to analyse the treatment impact. To evaluate distribution inclination, channel plots were built for every result. No noteworthy distribution predisposition was identified for any investigation result utilizing either measurable strategy. Furthermore, affectability examinations were directed by barring each in- vestigation thusly, to assess its relative impact on the pooled evaluation $[3,6-8,11,15]$.

\section{Results}

A total of 51 studies were identified and inclusion criteria was applied as illustrated in figure 1. After careful evaluation of titles, abstract, full text analysis and screening 5 studies were found to meet the inclusion criteria and were included for meta-analysis. A study which has a total of 1481 patients, out of which 807 patients underwent LACS and 674 underwent RACS. Another study was taken where 18 were right hemicolectomy, 1 sigmoidectomy, 3 abdominoperineal resection, 3 low anterior resection and 4 concomitant liver resections. Both the studies were used to compare Robotic and Laparoscopic methods of 2 different set of patients. [7,8, 23,24] Comparison of operating time, estimated blood loss, hospital stay, probability of complication, probability of anastomic leak and mortality for various patients is demonstrated in Figure 2. Calm measurement data and traits of the 2 clusters are seemed Table 1 . There were no tremendous differences between the social events to the extent age, BMI, or history of stomach medicinal methodology.

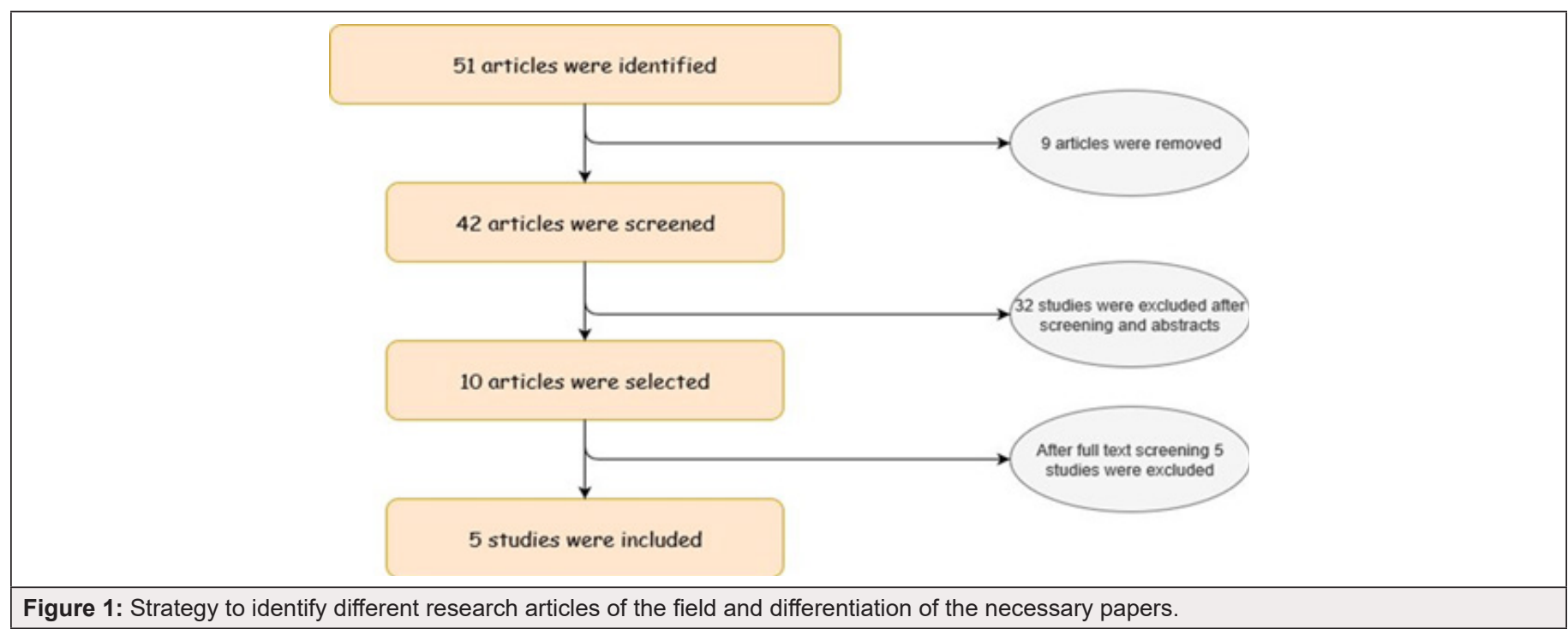

\begin{tabular}{|c|c|c|}
\hline \multicolumn{2}{|c|}{ Table 1: Comparing robotic and laparoscopic surgeries with age, gender, BMI, Diagnosis, Type of operation and Other medical diseases. } \\
\hline Laparoscopic (n=25) & Robotic (n=15) & $61.1(8.5) 0.997$ \\
\hline Age (y) & $61.1(10.7)$ & $13(86.7) 0.026$ \\
\hline Male [n (\%)] & $13(52.0)$ & $46.2(4.2) 0.158$ \\
\hline Body mass index & $28.9(6.3)$ & $4(26.7) 0.273$ \\
\hline Previous abdominal surgery. [n (\%)] & $11(44.0) 0.303$ & $7(60.0)$ \\
\hline Diagnosis. [n (\%)] & $14(56.0)$ & $2(13.0)$ \\
\hline Malignant disease of colon & $6(36.0)$ & $2(13.0)$ \\
\hline Polyps & $2(8.0)$ & \\
\hline Diverticular disease & $3(12.0)$ & 7 \\
\hline Other & & \\
\hline Types of operations (n) & 18 & 2 \\
\hline Right hemicolectomy & 0 & \\
\hline Left hemicolectomy & & \\
\hline
\end{tabular}




\begin{tabular}{|c|l|l|}
\hline Sigmoidectomy & 1 & 0 \\
\hline Total colectomy & 0 & 0 \\
\hline Abdominoperineal reaction & 3 & 1 \\
\hline Low anterior resection & 3 & 5 \\
\hline Concomitant liver resection & 4 & 0 \\
\hline
\end{tabular}

The mean time of patients encountering laparoscopic restorative system versus mechanical therapeutic method was $61.1 \pm$ 10.7 years versus $61.1 \pm 8.5$ years $(\mathrm{P}=.997)$, the mean BMI was $28.9 \pm 6.3$ versus $26.2 \pm 4.2(\mathrm{P}=.158)$, and the rate with a past loaded up with stomach medicinal technique was $44.0 \%$ versus $26.7 \%$ $(\mathrm{P}=.273)$. There was a basic qualification to the extent sex, with more patients being male and encountering mechanized therapeu- tic method $(86.7 \%$ versus $52.0 \%, \mathrm{P}=.026)$. There was no significant qualification in cautious sign between the 2 social occasions $(\mathrm{P}=.303)[1,7,8,25,26]$. Eighteen patients experienced right hemicolectomy, 1 encountered a sigmoidectomy, 3 experienced abdominoperineal resection (APR), and 3 experienced low front resection (LAR) performed laparoscopically.
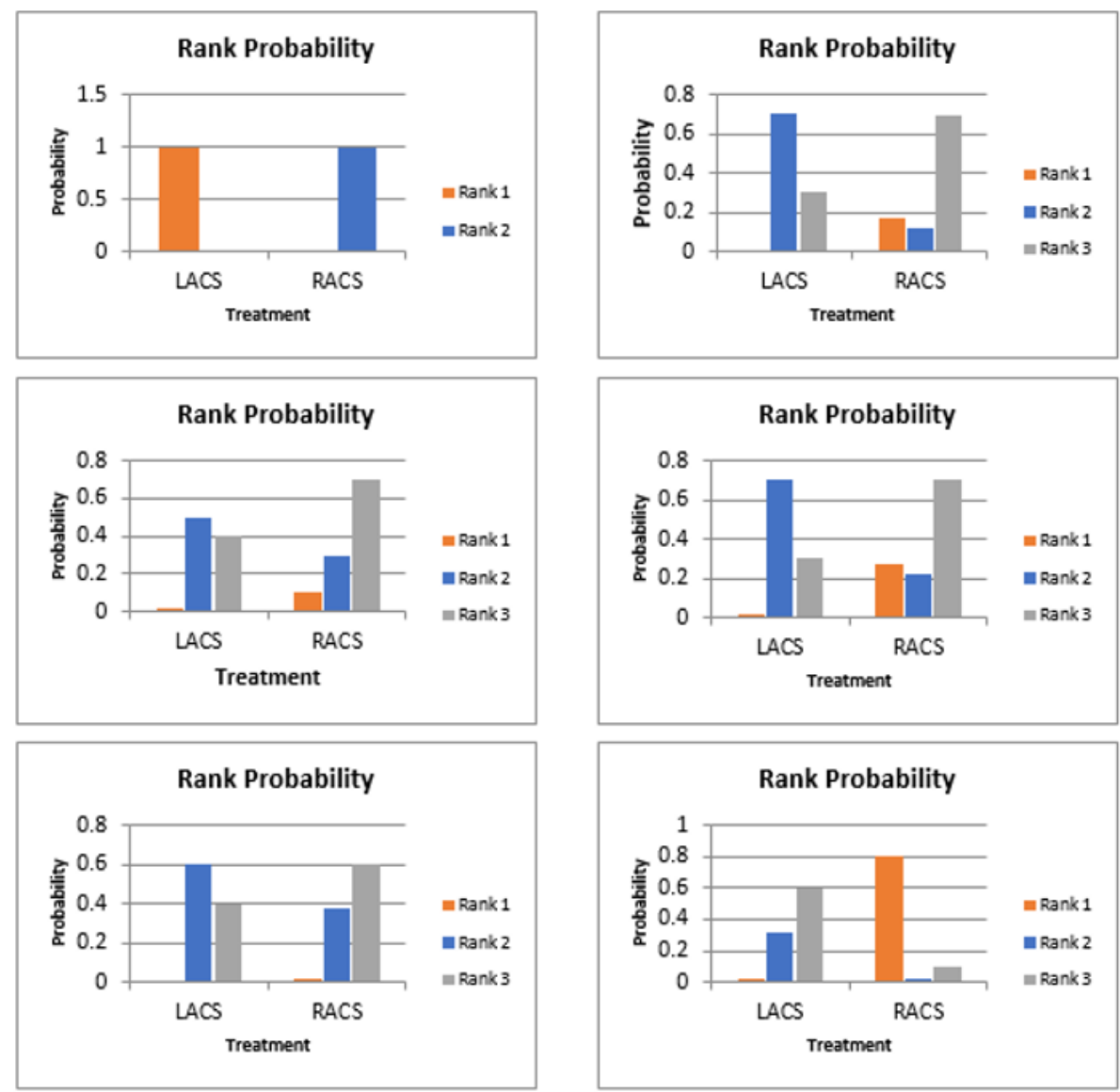

Figure 2: (A) Results of rank probability for operation time (B) Results of rank probability for estimated blood loss (C) Results of rank probability for length of hospital stay (D) Results of rank of probability for complication (E) Results of probability of mortality (F) Results of probability of anatomic leak LACS- Laparoscopic assisted colorectal surgery RACS- Robotic assisted colorectal surgery.

\section{Meta-Analysis}

\section{Estimated operative time}

The value of operative time ranged between 1.00 to 1.01 good iterative effect and completely stable results. The result of meta-analysis presented in Figure 2(A) revealed that almost both laparoscopic and robotic surgery takes about the same amount of operative time with robotic taking a little more amount of time comparatively. $[3,7,8,25,27]$ Although the relative difference between both is almost negligible.

\section{Estimated blood loss}

The analysis blood loss ranged from 1.00 to 1.01 , proving nearly stable results as seen in Figure 2 (B). Result of meta-analysis reveals that comparative robotic surgery had more blood loss than laparoscopic surgery $[1,7,8,27,28]$.

\section{Estimated meta-analysis of length of hospital stay}

The model of length of clinic stay had total assembly, great iterative impact, and stable outcomes. The consequences of the me- 
ta-examination uncovered that patients who experienced RACS had the briefest length of emergency clinic remain as illustrated in Figure 2 (C) [2,7,13-15].

\section{Estimated rate of complications}

The Figure 2 (D) suggests that the consequences of the meta-investigation uncovered that the inconvenience rate in patients who experienced RACS was the least, yet there was no huge contrast. $[3,6,7,28]$.

\section{Estimated analysis of mortality}

The analysis of mortality went from 1.00 to 1.01, demonstrating total intermingling, great iterative impact, and stable consequences of the model as seen in Figure 2 (E). The outcomes demonstrated that the death rate in patients who experienced RACS was the least, yet there was no noteworthy distinction contrasted and those in patients who experienced LACS. $[1,7,8,13,15]$.

\section{Estimated analysis of anastomotic leak}

The analysis of anastomotic spillage went from 1.00 to 1.01 , showing total assembly, great iterative impact, and stable conse- quence of the model. Figure 2 (F) suggests these outcomes uncovered that the rate of anastomotic spillage in patients who experienced LACS was the least; however, there was no huge contrast contrasted and those in patients who experienced RACS $[1,3,7,8]$.

\section{Estimated analysis of wound infection and bleeding}

The results revealed that the rate of wound infection in patients who underwent LACS was the least, but there was no significant difference compared with that in patients who underwent RACS. The result demonstrates that the rate of seeping in patients who experienced RACS was the least, yet there was no noteworthy contrast contrasted and those in patients who experienced LACS. The oncologic qualities of the colorectal undertakings performed for tumour resection were moreover noted (Figure 3). There was no tremendous difference in tumour sort out or histologic assessment between the 2 social occasions $[1,7,8,22,23,26]$. Furthermore, there were no basic differentiation in the amount of lymph centre points harvested between the laparoscopic gathering and the robotic surgery. No resections performed for damage yielded positive edges in either assembling.

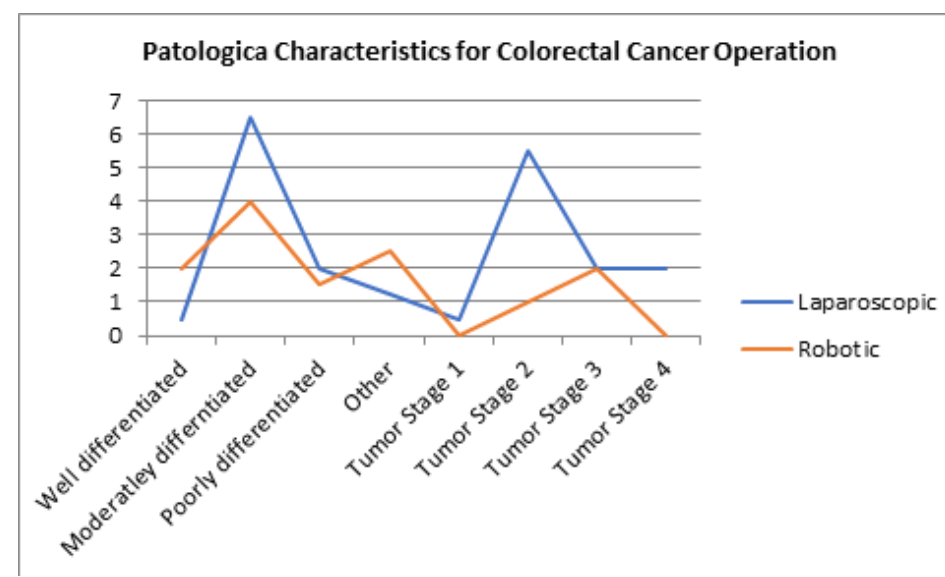

Figure 3: Comparing laparoscopic and robotic surgery for different types and stages of tumor.

\section{Discussions}

A network meta-analysis is performed comparing the curative effects of LACS and RACS in this study. Laparoscopic surgery has been widely accepted over open surgery for colorectal cancer. LACS has shown benefits and is also safer comparatively. For example, patients that have underwent laparoscopy surgery for colorectal cancer have demonstrated that it can yield a diminished length of hospital stay, oncologically sufficient resection, and no distinctions in postoperative stay $[1,6,7,10,24]$. In view of concentrates like these, laparoscopy is presently viewed as an adequate option in contrast to an open surgery in colorectal resection. However, conversion to open surgery still stays as a big pitfall for LACS. With the advent of new technology, RACS is now the growing field in medicine and is widely accepted for surgery in bariatric, urology, gynaecology and various other fields. $[8,11,13,17,26]$. The prevalence of RACS, one of the most recent advancements in laparoscopic medical procedure, has been expanding since it was first performed in cholecystectomy in 2001(Table 2).

The careful method is improved by the properties of the robot framework, for example, able to use both hands ability, 3-dimensional view, and tremor elimination. Similar to our examination, past investigations announced that RACS had the longest activity time $[1,3,9,24,26]$. However, it is important that the task time of RACS is negligible in progressively complex pelvic processes. However, the self-sufficiency of RACS is superior to that of LACS. A past report referenced that the expense of RACS was a lot higher than that of LACS. Also, it has been proposed that the length of medical procedure in the RACS was longer than that in the LACS gathering, while the quantity of lymph nodes collected, resection edge freedom, postoperative agony score, careful difficulties, and clinic stay were similar. The task times were accounted for to be altogether longer in patients treated with robots than that treated with lap- 
aroscopy, though there were no contrasts between the 2 bunches as to entanglements and emergency clinic stay, which was like our outcomes. Two arrangement looking at RACS and LACS in right col- ectomy have shown that RACS has a more extended case time and higher all out medical clinic cost than LACS however comparative evaluated blood loss and length of emergency clinic stay.

\begin{tabular}{|c|c|c|c|c|c|c|}
\hline Reference & Surgery & $\begin{array}{c}\text { Number of } \\
\text { patients }\end{array}$ & $\begin{array}{c}\text { Conversion } \\
\text { Rate }\end{array}$ & $\begin{array}{l}\text { Positive Circumferential } \\
\text { resection Margin }\end{array}$ & $\begin{array}{c}\text { Operative time, } \\
\text { minutes }\end{array}$ & $\begin{array}{c}\text { Complication } \\
\text { rate }\end{array}$ \\
\hline \multirow{2}{*}{ Bianchiet al.58 (2011) } & \multirow{2}{*}{ Robot Lap } & 25 & $0 \%$ & $0 \%$ & 240 & $16 \%$ \\
\hline & & 25 & $4 \%$ & $4 \%$ & 237 & $24 \%$ \\
\hline \multirow{2}{*}{ D’Annibaleet al.59 (2013) } & \multirow{2}{*}{ Robot Lap } & 50 & $0 \%$ & $0 \%$ & 270 & $10 \%$ \\
\hline & & 50 & $12 \%$ & $12 \%$ & 280 & $22 \%$ \\
\hline \multirow{2}{*}{ Ghezziet al.60 (2014) } & Robot & 65 & $1.50 \%$ & $0 \%$ & 299 & $41.50 \%$ \\
\hline & Open & 109 & - & $1.80 \%$ & 207.5 & $41.30 \%$ \\
\hline \multirow{2}{*}{ Yamagucheif al.61 (2015) } & Robot & 203 & $0 \%$ & $0 \%$ & $232.0+72$ & $8.90 \%$ \\
\hline & Lap & 239 & $3.30 \%$ & $1 \%$ & $227.6+-62.6$ & $34 \%$ \\
\hline Kimet al.62 & Robot & 33 & $6.10 \%$ & $16.10 \%$ & 441 & $45.60 \%$ \\
\hline-2015 & Lap & 66 & $0 \%$ & $6.70 \%$ & 227 & $39.40 \%$ \\
\hline \multirow{2}{*}{ Choetal.63 } & Robot & 278 & $0.40 \%$ & $5 \%$ & $361.6+91.9$ & $25.90 \%$ \\
\hline & Lap & 278 & $0.70 \%$ & $4.70 \%$ & $272.4 .+83.8$ & $23.70 \%$ \\
\hline Allemannet al.64 & Robot & 20 & $5 \%$ & $10 \%$ & 291 & $40 \%$ \\
\hline-2015 & Lap & 40 & $20 \%$ & $25 \%$ & 313 & $35 \%$ \\
\hline
\end{tabular}

It isn't vital for RACS and LACS to change over to the open approach. Although there was no huge clinical preferred standpoint for RACS in assessed blood loss, length of emergency clinic remains, and confusion rate contrasted and LACS, the lymph nodes around primary veins could be cleaned effectively dependent on the steady camera stage $[3,7-9,16,18,22]$. In addition, RACS gave solace to the specialist by giving a superior usable act. Also, the conversion rate to open was very low in RCS compared to LACS, which proves as an added benefit. An exceptionally intriguing investigation from Kang et al, contrasted three gatherings of patients and mid and low rectal tumours treated with either open, laparoscopic or robotic methodology $[14,21-23,27]$. They saw that the robotic gathering had a quicker postoperative recuperation with a lower emergency clinic remain, less agony and better example quality. The sickness free survival rate was comparative in all gatherings three years after medical procedure [29]. A noteworthy debilitation for specialist's keen on figuring out how to utilize a careful robot is the loss of touch vibe that is of huge significance in various methodology.

The activity of a specialist who can't feel the distinctive tissues and strain turns out to be significantly increasingly troublesome, and the dangers of aperture and damage increment. Furthermore, while "hand-sewn" procedures are all the more promptly performed with apply autonomy over laparoscopy, the tissue pressure on the sutures should be accumulated from obvious signals. $[7,8,13,28]$ These hindrances likewise exist in conventional laparoscopic methodologies and it is conceivable that new robotic innovation might almost certainly beat them. Despite the fact that this meta-analysis is extensive and the most present assessment of robotic and laparoscopic ways to deal with colon and rectal medical procedure to date, it ought to be deciphered with regards to some limitations $[2-4,11]$. This has various ramifications on the information, including consequences for the usable time and perioperative entanglements. Second, the scarcity of current randomized controlled preliminaries further constrains the consequences of meta-analysis. [3] Besides, not the majority of the investigations provided details regarding all results inspected inside this examination, prompting varieties in the included example sizes and factual power between results. Each investigation has its own inclinations and restrictions, with various incorporation and prohibition criteria, changing signs for medical procedure, and distinctive sorts of included colorectal strategies. $[12,16,17,21]$.

\section{Conclusions}

Taking everything into account, the present system meta-analysis proposes that RACS may be a superior treatment for CRC. Robotic surgery would in general have longer operative time, less blood loss, and a lower rate of change to laparotomy compared to laparoscopic surgery. However, future examinations including forthcoming randomized controlled preliminaries and cost-adequacy is needed to decide if robotic surgery will have it say in colorectal techniques.

\section{Acknowledgement}

The author(s) declared that no grants were involved in supporting this work.

\section{References}

1. Jacobs M, Verdeja JC, Goldstein HS (1991) Minimally invasive colon resection (laparoscopic colectomy). Surgical laparoscopy \& endoscopy 1: 144-150. 
2. D’Annibale A, Pernazza G, Morpurgo E, Monsellato I, Pende V, et al (2010) Robotic right colon resection: evaluation of first 50 consecutive cases for malignant disease. Annals of surgical oncology 17: 2856-2862.

3. Ngu JC, Tsang CB, Koh DC. (2017) The da Vinci Xi: a review of its capabilities, versatility, and potential role in robotic colorectal surgery. Robotic surgery 4: 77-85

4. Keller DS, Senagore AJ, Lawrence JK, Champagne BJ, Delaney CP (2014) Comparative effectiveness of laparoscopic versus robot-assisted colorectal resection. Surgical endoscopy 28: 212-221.

5. Alasari S, Min BS (2012) Robotic colorectal surgery: a systematic review. ISRN surgery 293894.

6. Challacombe B, Wheatstone S (2010) Telementoring and telerobotics in urological surgery. Current urology reports 11: 22-28.

7. Liao G, Zhao Z, Lin S et al. (2014) Robotic-assisted versus laparoscopic colorectal surgery: a meta-analysis of four randomized controlled trials. World journal of surgical oncology 12: 122.

8. Jimenez Rodriguez RM, Diaz Pavon JM, de La Portilla de Juan F, Prendes Sillero E, Hisnard Cadet Dussort JM, et al. (2011) [Prospective randomised study: robotic-assisted versus conventional laparoscopic surgery in colorectal cancer resection]. Cirugia espanola 89: 432-438.

9. Bertani E, Chiappa A, Biffi R, Bianchi PP, Radice D, et al. (2011)Assessing appropriateness for elective colorectal cancer surgery: clinical, oncological, and quality-of-life short-term outcomes employing different treatment approaches. International journal of colorectal disease. 26 1317-1327.

10. Yang Y, Wang F, Zhang P, Shi C, Zou Y, et al. (2012) Robot-assisted versus conventional laparoscopic surgery for colorectal disease, focusing on rectal cancer: a meta-analysis. Annals of surgical oncology 19: 3727 3736.

11. Liao G, Chen J, Ren C, Li R, Du S, et al. (2013) Robotic versus open gastrectomy for gastric cancer: a meta-analysis. PloS one 8: e81946.

12. Bhangu A, Brown G, Akmal M, Tekkis P (2012) Outcome of abdominosacral resection for locally advanced primary and recurrent rectal cancer. The British journal of surgery 99: 1453-1461.

13. Isik O, Gorgun E (2015) How Has the Robot Contributed to Colon Cancer Surgery? Clinics in colon and rectal surgery 28: 220-227.

14. Ma Y, Yang Z, Qin H, Wang Y (2011) A meta-analysis of laparoscopy compared with open colorectal resection for colorectal cancer. Medical oncology 28: 925-933.

15. Spinoglio G, Summa M, Priora F, quarati R, Testa S (2008) Robotic colorectal surgery: first 50 cases experience. Diseases of the colon and rectum 51: 1627-1632.

16. Trinh BB, Hauch AT, Buell JF, Kandil E (2014) Robot-assisted versus standard laparoscopic colorectal surgery. JSLS : Journal of the Society of Laparoendoscopic Surgeons 18(4): e2014.00154.
17. Trinh BB, Jackson NR, Hauch AT, Hu T, Kandil E (2014) Robotic versus laparoscopic colorectal surgery. JSLS : Journal of the Society of Laparoendoscopic Surgeons 18(4): e2014.00187.

18. SS T, F H, AL R, DL C (2010) Robotic Surgery of the Colon: The Peoria Experience. In: Baik SH, ed. Robot Surgery. Online: InTech.

19. Ng KH, Lim YK, Ho KS, Ooi BS, Eu KW (2009) Robotic-assisted surgery for low rectal dissection: from better views to better outcome. Singapore medical journal 50: 763-767.

20. Luca F, Cenciarelli S, Valvo M, Pozzi S, Faso FL, et al. (2009) Full robotic left colon and rectal cancer resection: technique and early outcome. Annals of surgical oncology 16: 1274-1278.

21. Scandola M, Grespan L, Vicentini M, Fiorini P (2011) Robot-assisted laparoscopic hysterectomy vs traditional laparoscopic hysterectomy: five metaanalyses. Journal of minimally invasive gynecology 18: 705-715.

22. Maeso S, Reza M, Mayol JA, Blasco JA, Guerra M, et al. (2010) Efficacy of the Da Vinci surgical system in abdominal surgery compared with that of laparoscopy: a systematic review and meta-analysis. Annals of surgery 252: 254-262.

23. Deutsch GB, Sathyanarayana SA, Gunabushanam V, Mishra N, Rubach E, et al. (2012) Robotic vs. laparoscopic colorectal surgery: an institutional experience. Surgical endoscopy 26: 956-963.

24. Patel CB, Ragupathi M, Ramos-Valadez DI, Haas EM (2011) A three-arm (laparoscopic, hand-assisted, and robotic) matched-case analysis of intraoperative and postoperative outcomes in minimally invasive colorectal surgery. Diseases of the colon and rectum 54: 144-150.

25. Kim CW, Kim CH, Baik SH (2014) Outcomes of robotic-assisted colorectal surgery compared with laparoscopic and open surgery: a systematic review. Journal of gastrointestinal surgery : official journal of the Society for Surgery of the Alimentary Tract 18: 816-830.

26. Poon JT, Law WL (2009) Laparoscopic resection for rectal cancer: a review. Annals of surgical oncology 16: 3038-3047.

27. Morner ME, Gunnarsson U, Jestin P, Svanfeldt M (2012) The importance of blood loss during colon cancer surgery for long-term survival: an epidemiological study based on a population-based register. Annals of surgery 255: 1126-1128.

28. Amato A, Pescatori M (2006) Perioperative blood transfusions for the recurrence of colorectal cancer. The Cochrane database of systematic reviews CD005033.

29. Baek SJ, Kim SH, Cho JS, Shin JW, Kim J (2012) Robotic versus conventional laparoscopic surgery for rectal cancer: a cost analysis from a single institute in Korea. World journal of surgery 36: 2722-2729. 